\title{
Generalized quantum dimer model applied to the frustrated Heisenberg model on the square lattice: Emergence of a mixed columnar-plaquette phase
}

\author{
A. Ralko, ${ }^{1}$ M. Mambrini, ${ }^{2}$ and D. Poilblanc ${ }^{2}$ \\ ${ }^{1}$ Institut Néel, CNRS and Université de Grenoble, F-38000 Grenoble, France \\ ${ }^{2}$ Laboratoire de Physique Théorique, CNRS and Université de Toulouse, F-31062 Toulouse, France \\ (Received 13 May 2009; revised manuscript received 31 August 2009; published 30 November 2009)
}

\begin{abstract}
Aiming to describe frustrated quantum magnets with nonmagnetic singlet ground states, we have extended the Rokhsar-Kivelson (RK) loop expansion to derive a generalized quantum dimer model containing only connected terms up to arbitrary order. For the square-lattice frustrated Heisenberg antiferromagnet $\left(J_{1}-J_{2}-J_{3}\right.$ model), an expansion up to eighth order shows that the leading correction to the original RK model comes from dimer moves on length-6 loops. This model free of the original sign problem is treated by advanced numerical techniques. The results suggest that a rotationally anisotropic plaquette phase [A. Ralko, D. Poilblanc, and R. Moessner, Phys. Rev. Lett. 100, 037201 (2008)] is the ground state of the Heisenberg model in the parameter region of maximum frustration.
\end{abstract}

DOI: 10.1103/PhysRevB.80.184427

\section{INTRODUCTION}

Over the last decades theoretical efforts have been devoted to study quantum phases of two-dimensional frustrated quantum magnets, motivated by the discovery of experimental antiferromagnets showing the absence of long-range magnetic ordering down to very low temperatures. ${ }^{1-5}$ In such systems a gap to magnetic excitation traditionally opens up while the spin-SU(2) symmetry remains unbroken. Two classes of "singlet" phases have been distinguished. Valencebond crystals (VBC) where some spatial symmetries are spontaneously broken and spin liquid for which all symmetries remain unbroken (e.g., the resonating valence-bond liquid. $\left.{ }^{6}\right)$.

However, it is usually difficult to characterize the singlet phases in simple microscopic $S=1 / 2$ models. For example, in the well-known $J_{1}-J_{2}$ Heisenberg $S=1 / 2$ antiferromagnet on the square lattice, where frustration is controlled by the next-nearest interaction $J_{2}$, no definitive answer has been given on the nature of the nonmagnetic Ground State (GS) for maximal frustration at $J_{2} / J_{1} \sim 0.5$. Mambrini et $\mathrm{al}^{7}{ }^{\mathrm{re}} \mathrm{re}$ cently addressed a work to this task, studying the $J_{1}-J_{2}-J_{3}$ model, containing an extra next-next-nearest neighbor $J_{3}$ frustrating antiferromagnetic,
PACS number(s): 75.10.Jm, 05.30. $-\mathrm{d}, 05.50 .+\mathrm{q}$

$$
\mathcal{H}=J_{1} \sum_{\langle i, j\rangle} \mathbf{S}_{i} \cdot \mathbf{S}_{j}+J_{2} \sum_{\langle\langle i, j\rangle\rangle} \mathbf{S}_{i} \cdot \mathbf{S}_{j}+J_{3} \sum_{\langle\langle i, j\rangle\rangle\rangle} \mathbf{S}_{i} \cdot \mathbf{S}_{j} .
$$

Interestingly, in this model the description in terms of nearest-neighbor valence-bond (NN VB) coverings ${ }^{8}$ is excellent in some extended region of parameter space, in particular, around the point $J_{2}=J_{3}=1 / 4$, with some significant extension along the line $\left(J_{2}+J_{3}\right)=J_{1} / 2$. This model is therefore one simple canonical case where a truncation within the nearest-neighbor singlet configuration basis is legitimate and can be used as a simpler and convenient framework.

In this paper, we have extended the Rokhsar-Kivelson (RK) loop expansion to derive a generalized quantum dimer model (QDM) acting in the space of hardcore $N N$ dimer coverings of the lattice. We show that this expansion based on the hierarchy of the overlap matrix elements between the dimer coverings leads to an effective Hamiltonian that contains a sum of dimer moves, each involving only a single closed loop or loops at finite distances (connected term). In other words, all disconnected terms cancel out order by order. We apply this procedure to the $J_{1}-J_{2}-J_{3}$ model and show that the leading contributions are of the form of a simple generalized QDM on the square lattice which, in addition to original QDM, ${ }^{9}$ contains an additional loop-6 term which brings kinetic competition in the system. The effective Hamiltonian then reads

$$
\mathcal{H}^{\mathrm{eff}}=v \sum_{p} \square \square_{p}-t_{4} \sum_{p} \square \square_{p}-t_{6} \sum_{p} \square \square .
$$

where the sums run, respectively, over all square or rectangular plaquettes of the square lattice. Here we use the following convention: (i) white plaquettes denote kinetic (offdiagonal) operators that flip dimers around the thick contour and (ii) yellow plaquettes stand for potential (diagonal) operators that leave configurations unchanged with a factor 1 if it is flippable around the thick contour and 0 in the opposite case. In the following, the omission of $p$ indices in the diagrammatic notation is a shortcut notation for an implicit summation over all inequivalent plaquettes with a given shape. For example,

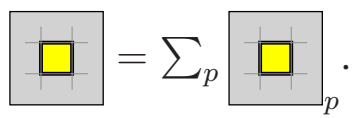


TABLE I. (Color online) Expansion of $\mathcal{O}, \mathcal{H}$, and $\mathcal{H}^{\text {eff }}$ up to order $\alpha^{6}$. Note that the effective Hamiltonian contains only local (connected) processes. Some processes (marked as $\emptyset$ ) does not appear in $\mathcal{O}$ or $\mathcal{H}$ but are produced in $\mathcal{H}^{\text {eff }}$ by contractions of the generically noncommuting terms of the expansion (see Appendix $\mathrm{B}$ for details).

\begin{tabular}{|c|c|c|c|}
\hline \hline Processes & $\mathcal{O}$ & $\mathcal{H}$ & $\mathcal{H}^{\text {eff }}=\mathcal{O}^{-1 / 2} \mathcal{H} \mathcal{O}^{-1 / 2}$ \\
\hline Id & 1 & 0 & 0 \\
\hline$\square$ & $\emptyset$ & $\emptyset$ & $2\left(J_{1}-J_{2}\right) \alpha^{4}$ \\
\hline$\square$ & $\alpha^{2}$ & $2\left(-J_{1}+J_{2}\right) \alpha^{2}$ & $-2\left(J_{1}-J_{2}\right) \alpha^{2}\left(1+\alpha^{4}\right)$ \\
\hline$\square$ & $\alpha^{4}$ & $2\left(-2 J_{1}+2 J_{2}+J_{3}\right) \alpha^{4}$ & $2\left(-J_{1}+J_{2}+J_{3}\right) \alpha^{4}$ \\
\hline$\square \square$ & $\alpha^{4}$ & $4\left(-J_{1}+J_{2}\right) \alpha^{4}$ & 0 \\
\hline$\square \square$ & $\alpha^{6}$ & $2\left(-3 J_{1}+3 J_{2}+J_{3}\right) \alpha^{6}$ & 0 \\
\hline$\square$ & $\alpha^{6}$ & $\left(-6 J_{1}+7 J_{2}+4 J_{3}\right) \alpha^{6}$ & $\left(-2 J_{1}+3 J_{2}+2 J_{3}\right) \alpha^{6}$ \\
\hline$\square$ & $\alpha^{6}$ & $2\left(-3 J_{1}+3 J_{2}+2 J_{3}\right) \alpha^{6}$ & $\left(-J_{1}+J_{2}+2 J_{3}\right) \alpha^{6}$ \\
\hline$\square$ & $\alpha^{6}$ & $6\left(-J_{1}+J_{2}\right) \alpha^{6}$ & 0 \\
\hline$\square$ & $\emptyset$ & $\emptyset$ & $\left(J_{1}-J_{2}-J_{3}\right) \alpha^{6}$ \\
\hline$\square$ & \multicolumn{2}{|l|}{$\emptyset$} \\
\hline$\square$
\end{tabular}

Guided by a variational approach and combining numerical techniques such as exact diagonalizations (ED) and zerotemperature Green's-function Monte Carlo, we compute the phase diagram of this model. More specifically, we give evidence in favor of a mixed columnar-plaquette phase first proposed in Ref. 10 and, since, evidenced in number of other contexts. ${ }^{11}$ Remarkably, this phase is found to be stable even in the presence of the loop-6 fluctuations. Hereafter, using the relation between the effective and microscopic models, we argue in favor of the SU(2)-invariant version (i.e., applicable to a spin- $1 / 2$ model instead of a dimer model) of the above mixed columnar-plaquette phase in the $J_{1}-J_{2}-J_{3}$ microscopic model along the maximally frustrated line $J_{2}+J_{3}$ $=J_{1} / 2$ where an approach restricting to the short-range VB basis has been justified previously. ${ }^{7}$

\section{DERIVATION OF THE MODEL}

A systematic way to derive the generalized QDM Hamiltonian (1) consists of (i) projecting the Heisenberg model in the manifold formed by NN VB coverings of the square lattice and (ii) perform a transformation that turns the nonorthogonal VB basis into the orthogonal quantum dimer basis. The key ingredient of the calculation is the overlap matrix $\mathcal{O}_{\varphi, \psi}=\langle\varphi \mid \psi\rangle$, where $|\varphi\rangle$ and $|\psi\rangle$ are two NN VB states. Step (i) is equivalent to solving $\mathcal{H}|\phi\rangle=E|\phi\rangle$, where $|\phi\rangle$ is re- stricted to the NN VB subspace. Writing $|\phi\rangle=\sum_{i} \alpha_{i}\left|\varphi_{i}\right\rangle$, where the sum runs over the NN VB states $\left|\varphi_{i}\right\rangle$, the original eigenvalue problem turns into a generalized one $\Sigma_{j}\left\langle\varphi_{i}|\mathcal{H}| \varphi_{j}\right\rangle \alpha_{j}=E \Sigma_{j}\left\langle\varphi_{i} \mid \varphi_{j}\right\rangle \alpha_{j}$. In turn, this problem is equivalent to the conventional eigenvalue problem $\mathcal{H}^{\text {eff }}|\phi\rangle=E|\phi\rangle$ with $\mathcal{H}^{\text {eff }}=\mathcal{O}^{-1 / 2} \mathcal{H O}^{-1 / 2}$ which provides the transformation required by step (ii).

Using a convention where all bond singlets are oriented from sites $A$ to sites $B$ according to the canonical bipartition of the square lattice, the overlap matrix can be written as $\mathcal{O}_{\varphi, \psi}=\alpha^{N-2 n_{l}(\varphi, \psi)}$, where $N$ is the size of the system, $n_{l}(\varphi, \psi)$ are the number of loops in the overlap diagram obtained by superimposing the two configurations, and $\alpha=1 / \sqrt{2}$. On the other hand, $\left\langle\varphi\left|\mathbf{S}_{i} . \mathbf{S}_{j}\right| \psi\right\rangle=\varepsilon\langle\varphi \mid \psi\rangle$ with $\varepsilon=-3 / 4$ (respectively, $\varepsilon=+3 / 4)$ if $i$ and $j$ belongs to the same loop of the overlap diagram but belong to distinct sublattices (respectively, belong to the same sublattice) and $\varepsilon=0$ if $i$ and $j$ belongs to two distinct loops. Using a convenient scaling and shifting $\mathcal{H}$ $\rightarrow(4 / 3) \mathcal{H}+J_{1} N / 2$ of Hamiltonian (1), the matrix elements $\langle\varphi|\mathcal{H}| \psi\rangle$ can be expressed as $\mathcal{H}_{\varphi, \psi}=h_{\varphi, \psi} \mathcal{O}_{\varphi, \psi}$, where $h_{\varphi, \psi}$ only depends on the loops configuration. In particular, this convention enforces $h_{\varphi, \varphi}=0$ for all $\varphi$.

It is then possible to expand $\mathcal{O}$ and $\mathcal{H}$ in powers of $\alpha$ and compute $\mathcal{H}^{\text {eff }}=\mathcal{O}^{-1 / 2} \mathcal{H O}^{-1 / 2}$ accordingly as shown in Table I up to $\alpha^{6}$. The expansion up to $\alpha^{8}$ as well further technical details of the calculation are given in the Appendix B. It is 


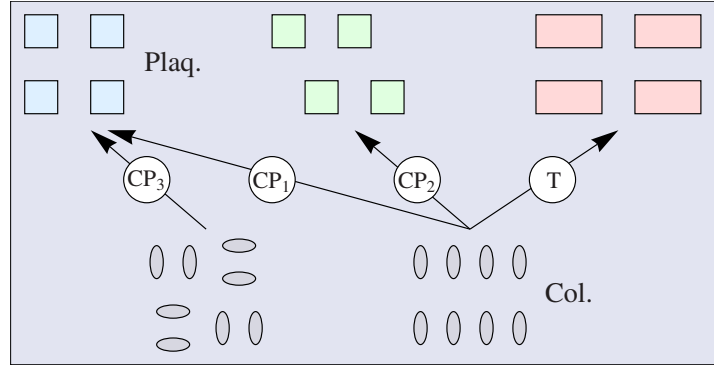

FIG. 1. (Color online). VBC states considered in this work. Generalized anisotropic VBC states labeled by $\mathrm{CP}_{a}$ (mixed columnar-plaquette phases) and $T$ (trimerized phase) interpolate between the most symmetric limits shown in the figure.

worth mentioning two peculiarities of this expansion: (i) contrary to several previous approaches $\mathrm{s}^{9,12,13}$ our expansion is not controlled by the length of the loops but by the actual amplitudes of the overlap matrix elements that only depend on the overall number of loops in the overlap diagrams and (ii) all nonlocal and disconnected processes appearing in both $\mathcal{H}$ and $\mathcal{O}$ cancel in the expression of $\mathcal{H}^{\text {eff }}$.

Let us discuss the results of this expansion. When truncated up to order $\alpha^{2}$, we recover the usual Hamiltonian obtained in Ref. 9 with $v /\left|t_{4}\right|=\alpha^{2}=1 / 2$. Note that such a drastic truncation appears a bit pathological in the sense that it does not capture any aspect of the frustration of the original model, $v /\left|t_{4}\right|$ is indeed independent of $J_{2} / J_{1}$. In the perspective of a justification of QDM model from the Heisenberg model, nontrivial effects emerge from order $\alpha^{4}$. Furthermore, considering the last column of Table I it is quite easy to see that, in the maximally frustrated region of the phase diagram $\left(J_{2}+J_{3} \sim J_{1} / 2\right)$, where the validity of the NN VB approach have been established, ${ }^{7}$ the three processes retained in Eq. (2) are larger by at least a factor of 2 compared to the higherorder kinetic processes (see Table IV). Importantly, we find that $t_{4}>0$ and $t_{6}>0$ which enable the use of efficient stochastic methods not applicable to the original frustrated spin model which suffers from the so-called "minus sign" problem. Note that the next-leading corrections are diagonal terms (see Table IV) and, hence, could also be added in future developments.

\section{VARIATIONAL ANALYSIS}

We now turn to the investigation of the effective Hamiltonian (2). We start with some discussion of the expected VBC phases shown in Fig. 1. Regular columnar and plaquette phases have been introduced in the context of the frustrated $J_{1}-J_{2}$ model and of the QDM. ${ }^{14}$ More recently, an anisotropic mixed columnar-plaquette phase has been introduced..$^{10}$ We consider here the possibility of such phases which interpolate between the simple higher-symmetry VBC (such as columnar or plaquette). Because of the presence of loop-6 dimer moves, we also consider the possibility of a trimerization of the columns of dimers. We summarize the quantum numbers of the degenerate GS of the various VBC in Table II. This will be used further in this paper to analyze the low-energy spectrum of Hamiltonian (2).
TABLE II. Quantum numbers of the eigenstates collapsing toward the same degenerate GS for each of the ordered phases considered in this paper. When applicable, we used the standard notations for the irreducible representations of the $C_{4 v}$ and $C_{2 v}$ point groups, whose elements are defined w.r.t. a plaquette center. Definitions of the $\Gamma, M$, and $K$ points in the Brillouin zone are given in Fig. 2. $(\times 2)$ denotes an additional first-excited level (denoted by * in the text) in the $\left(M, A_{1}\right)$ sector. The states with momenta $Q_{B}$ $=( \pm 2 \pi / 3,0), \quad(0, \pm 2 \pi / 3), \quad(\pi, \pm 2 \pi / 3), \quad(\pi, \pm 2 \pi / 3), \quad Q_{2}$ $=( \pm \pi / 2, \pi),(\pi, \pm \pi / 2)$, and $Q_{3}=( \pm \pi / 2, \pm \pi / 2)$ are even under reflection w.r.t. the momentum directions. The degeneracy of the pure columnar or plaquette (mixed) phases is 4 (8) and it is 12 for the trimerized phase.

\begin{tabular}{lcccccccc}
\hline \hline & $\Gamma, A_{1}$ & $\Gamma, B_{1}$ & $M, A_{1}$ & $K, A_{1}$ & $K, B_{1}$ & $Q_{B}$ & $Q_{2}$ & $Q_{3}$ \\
\hline Col. & $\sqrt{ }$ & $\sqrt{ }$ & $\sqrt{ }$ & & & & & \\
Pla. & $\sqrt{ }$ & & $\sqrt{ }$ & $\sqrt{ }$ & & & & \\
$\mathrm{CP}_{1}$ & $\sqrt{ }$ & $\sqrt{ }$ & $\sqrt{ }(\times 2)$ & $\sqrt{ }$ & $\sqrt{ }$ & & & \\
$\mathrm{CP}_{2}$ & $\sqrt{ }$ & $\sqrt{ }$ & $\sqrt{ }$ & & & & $\sqrt{ }$ & \\
$\mathrm{CP}_{3}$ & $\sqrt{ }$ & $\sqrt{ }$ & $\sqrt{ }$ & & & & & $\checkmark$ \\
$T$ & $\sqrt{ }$ & $\sqrt{ }$ & $\sqrt{ }$ & & & $\sqrt{ }$ & & \\
\hline \hline
\end{tabular}

Before showing the results of an extensive numerical analysis, we first start with a simple variational analysis. Indeed, variational ansatze for the VBC phases of Fig. 1 can be easily constructed as tensor products of resonating plaquette states (see Appendix A) and the knowledge of their relative stability provides a useful guide for the numerical search of VBC (but is also subject to some artifact of the variational method). For convenience, let us map the two-dimensional parameter space on a sphere by expressing the Hamiltonian parameters in terms of two Euler angles $\theta$ and $\phi$, as $v$ $=\cos (\phi) \sin (\theta), t_{4}=\cos (\phi) \cos (\theta)$, and $t_{6}=\sin (\phi)$. The variational phase diagram (Appendix A) in the $(\theta, \phi)$ plane con-
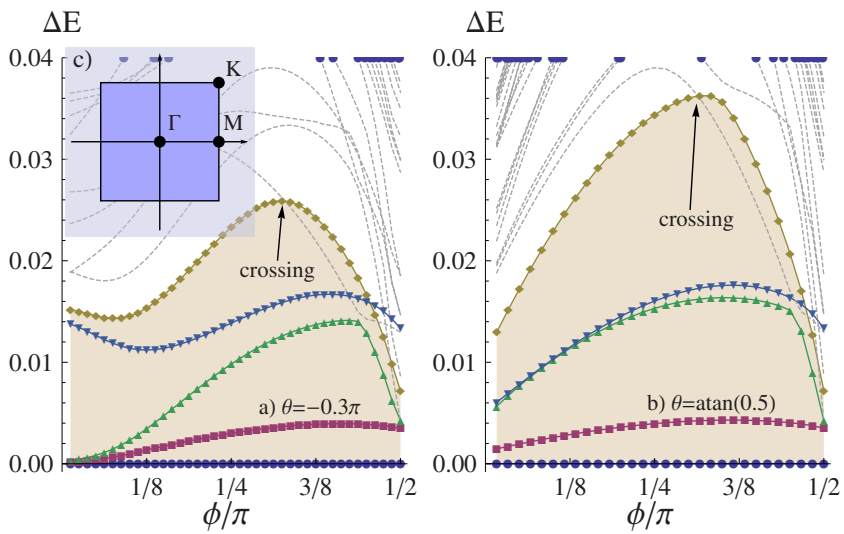

FIG. 2. (Color online). Typical ED low-energy spectra on a 8 $\times 8$ cluster (the GS energy is set to zero) for (a) $\theta=-0.3 \pi$ and (b) $\theta=\tan ^{-1}(0.5)$ as a function of $\phi$. Levels of special symmetries (see text) are highlighted as colored symbols, from bottom to top: $\left(\Gamma, A_{1}\right)$ (corresponding to the GS), $\left(M, A_{1}\right),\left(\Gamma, B_{1}\right),\left(K, A_{1}\right)$, and $\left(M, A_{1}\right)^{*}$. The arrow indicates the level crossing that marks the limit of the region where the latter levels correspond to the lowest excitations. Inset: Brillouin zone and its high-symmetry points $\Gamma$ $=(0,0), M=(\pi, 0)$, and $K=(\pi, \pi)$. 


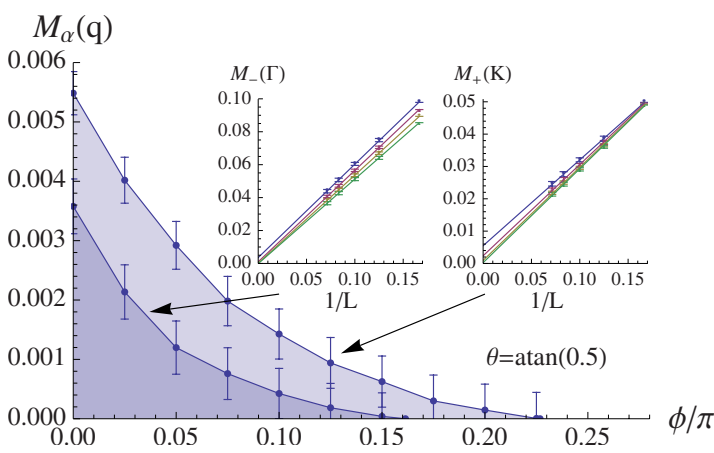

FIG. 3. (Color online). Extrapolations in the thermodynamic limit of the order parameters defined in the text characterizing the mixed $\mathrm{CP}_{1}$ columnar-plaquette phase. Insets: finite-size scaling of both $M_{+}(\pi, \pi)$ and $M_{-}(0,0)$ as a function of the inverse linear cluster size, using 36 sites $(6 \times 6), 64$ sites $(8 \times 8), 100$ sites $(10$ $\times 10), 144$ sites $(12 \times 12)$, and 196 sites $(14 \times 14)$ square clusters. The chosen value of $\theta$ corresponds to the case of the $J_{1}-J_{2}-J_{3}$ model studied in this work.

tains three phases, (i) a RK region, (ii) the well-known completely isotropic four-site plaquette phase, and (iii) a large domain covered by the trimerized $\mathrm{VBC}$ with a six-site unitcell interpolating between the columnar phase and the pure six-site plaquette phase. For zero loop-6 kinetic term $(\phi$ $=0)$, the phase diagram is the same than the one obtained in Ref. 11 (for $\theta>-0.25 \pi$ ). Once $\phi$ is turned on, kinetic fluctuations due to 6-loop kinetic terms suppress, at a given point, the standard four-site plaquette and RK phases. Nevertheless, the latter phases are rather robust under the additional $t_{6}$ kinetic term in the vicinity of the RK point (see thick lines in Fig. 5). However, the variational approach

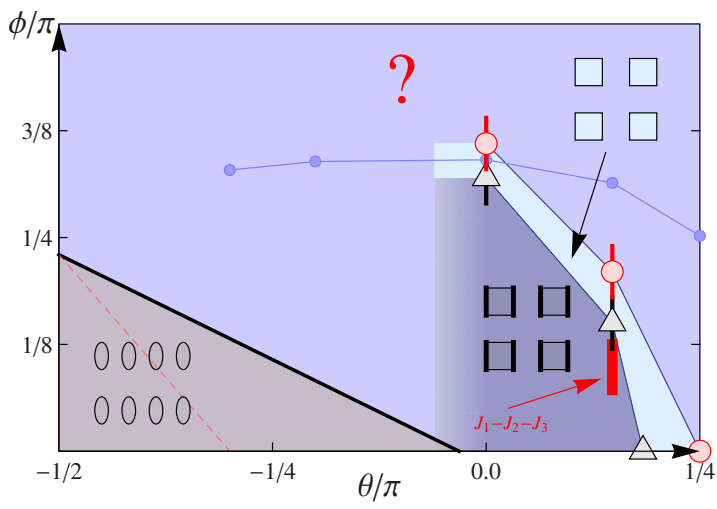

FIG. 4. (Color online). Phase diagram in the $(\theta, \phi)$ plane obtained from numerical simulations. The previous knowledge of the $\phi=0$ case (Ref. 10) has been used, in particular, to estimate the limit of the columnar phase as an approximate (black) straight line. The boundary of the pure plaquette $\left(\mathrm{CP}_{1}\right.$ mixed $)$ VBC phase obtained from the finite-size scaling of the associated order parameter is indicated by large circles (triangles). The (red) thick segment corresponds to the parameter region of the frustrated quantum antiferromagnet with $J_{2}+J_{3}=J_{1} / 2$ according to the mapping described in the text. Crude ED estimates of the boundaries of the columnar phase and of the $\mathrm{CP}_{1}$ phase are indicated by a thin dashed line and by small (blue) circles, respectively. The (light blue) region marked by a question mark has not been identified.

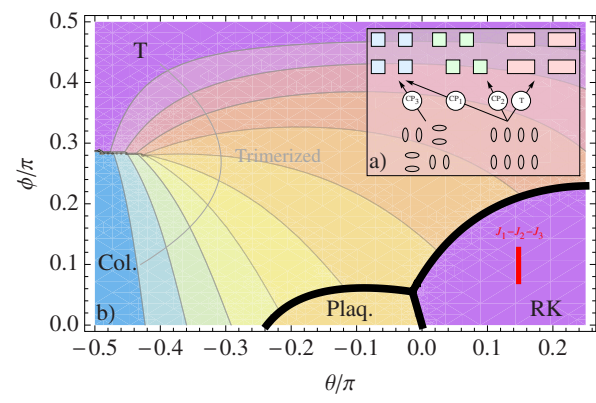

FIG. 5. (Color online) (a) Variational wave functions (associated to their respective VBC states) considered in this appendix. Generalized anisotropic VWF labeled by $\left|\mathrm{CP}_{a}\right\rangle$ and $|T\rangle$ interpolate between the most symmetric limits shown in the figure. (b) Variational phase diagram as a function of $\theta$ ( $x$ axis) and $\phi$ ( $y$ axis). The topographic map of $\alpha$ is depicted by the continuous gray lines. The line joining the Columnar phase and the trimerized one means that these regions are continuously connected in terms of $\alpha$. The thick red line shows the region of parameters of the effective model that maps to the $J_{1}-J_{2}-J_{3}$ model along the $J_{2}+J_{3}=J_{1} / 2$ line for which the ground state is well described by singlet coverings.

overestimate the stability of the RK phase (in fact limited to a single point with algebraic dimer correlations) and of the trimerized phase (the corresponding wave function has more four-site flippable plaquettes than plaquette counterpart). In contrast, no mixed anisotropic VBC phase is found. A careful numerical approach is therefore necessary.

\section{NUMERICAL RESULTS}

We now move to $\mathrm{ED}$ of clusters up to $8 \times 8$ sites. More especially, we compute the lowest-energy spectrum in each symmetry sectors, using both translations and point-group symmetries. Our analysis is based on the symmetry classification of the tour of states in the $(\theta, \phi)$ plane. In other words, each symmetry-breaking VBC phase is characterized by a finite degeneracy of the GS with a set of well-defined quantum numbers (see table) separated by a gap from the continuum. On a finite-size cluster, the degenerate GS is split but a close inspection of the low-energy spectrum can provide informations on the VBC phase (if the cluster is large enough). For $t_{6}=0$ (i.e., $\phi=0$ ), previous results (extrapolated to the thermodynamic limit) show that a phase transition between the columnar phase and a mixed columnar-plaquette phase (in fact the $\mathrm{CP}_{1}$ phase of this paper) occurs around $\theta$ $\sim-0.03 \pi$. Such a phase can be obtained via an in-phase spontaneous dimerization in the direction of the columns of dimers of the columnar phase or, equivalently, via a spontaneous rotation symmetry breaking of the pure plaquette phase. To simplify the discussion, we describe here two representative set of parameters that contain these two phases, $\theta=a \tan (0.5)\left(v / t_{4}=0.5\right)$ and $\theta=-0.3 \pi$, for which we have computed, as a function of $\phi$, the spectrum of the effective model by full ED. The spectra (defined w.r.t. the respective GS energies) are displayed in Fig. 2. Special symbols have been used to label five of the six low-energy levels (seven states over eight) associated to the mixed $\mathrm{CP}_{1}$ columnarplaquette phase. We do not consider the last one, the $\left(K, B_{1}\right)$ 
TABLE III. Expansions of $\mathcal{O}$ and $\mathcal{H}$ up to order $\alpha^{8}$.

\begin{tabular}{|c|c|c|}
\hline Processes & $\mathcal{O}$ & $\mathcal{H}$ \\
\hline Id & 1 & 0 \\
\hline & $\alpha^{2}$ & $2\left(J_{2}-J_{1}\right) \alpha^{2}$ \\
\hline$\square \square$ & $\alpha^{4}$ & $4\left(J_{2}-J_{1}\right) \alpha^{4}$ \\
\hline & $\alpha^{4}$ & $2\left(-2 J_{1}+2 J_{2}+J_{3}\right) \alpha^{4}$ \\
\hline$\square \square \square$ & $\alpha^{6}$ & $6\left(J_{2}-J_{1}\right) \alpha^{6}$ \\
\hline$\square \square$ & $\alpha^{6}$ & $2\left(-3 J_{1}+3 J_{2}+J_{3}\right) \alpha^{6}$ \\
\hline & $\alpha^{6}$ & $\left(-6 J_{1}+7 J_{2}+4 J_{3}\right) \alpha^{6}$ \\
\hline & $\alpha^{6}$ & $2\left(-3 J_{1}+3 J_{2}+2 J_{3}\right) \alpha^{6}$ \\
\hline$\square \square \square \square$ & $\alpha^{8}$ & $8\left(J_{2}-J_{1}\right) \alpha^{8}$ \\
\hline$\square \square \square$ & $\alpha^{8}$ & $2\left(-4 J_{1}+4 J_{2}+J_{3}\right) \alpha^{8}$ \\
\hline$\square \square$ & $\alpha^{8}$ & $4\left(-2 J_{1}+2 J_{2}+J_{3}\right) \alpha^{8}$ \\
\hline$\square \square \square$ & $\alpha^{8}$ & $4\left(-2 J_{1}+2 J_{2}+J_{3}\right) \alpha^{8}$ \\
\hline & $\alpha^{8}$ & $\left(-8 J_{1}+9 J_{2}+4 J_{3}\right) \alpha^{8}$ \\
\hline \begin{tabular}{|l|} 
\\
\end{tabular} & $\alpha^{8}$ & $2\left(-4 J_{1}+4 J_{2}+3 J_{3}\right) \alpha^{8}$ \\
\hline & $\alpha^{8}$ & $\left(-8 J_{1}+9 J_{2}+6 J_{3}\right) \alpha^{8}$ \\
\hline & $\alpha^{8}$ & $\left(-8 J_{1}+10 J_{2}+6 J_{3}\right) \alpha^{8}$ \\
\hline & $\alpha^{8}$ & $\left(-8 J_{1}+10 J_{2}+6 J_{3}\right) \alpha^{8}$ \\
\hline
\end{tabular}

level, which is believed to be more affected by finite-size effects. The plots show wide intervals of $\phi$, where the above five levels are the true lowest eigenenergies, hence pointing toward the mixed phase as a possible GS. The level crossing at which the low-energy spectrum becomes not anymore compatible with such a phase is indicated by an arrow. This level crossing can be used as a first crude estimator of the range of stability of the mixed phase. Surprisingly enough, the spectrum at $\theta>0$ is not drastically affected by a finite value of $t_{6}(\phi>0)$, showing that the mixed phase is rather stable w.r.t an extra loop- 6 term, up to $\phi \simeq 0.3 \pi$. This range of stability will be corroborated by our thermodynamic limit extrapolations of the order parameters (see below). In contrast, at $\theta=-0.3 \pi$ and small $\phi$, one can see that the very lowest levels of the spectrum (i.e., those really separated by a sizable gap from the rest) are compatible with the columnar phase whose symmetries are given in Table II. A narrow region of mixed columnar-plaquette phase might however exist at intermediate $\phi$ values before the level crossing involving the $\left(M, A_{1}^{*}\right)$ state occurs. To finish this ED analysis, let us mention that, apart from the pure columnar and the mixed $\mathrm{CP}_{1}$ columnar-plaquette phases, no region in parameter space could be found where the low-energy spectrum is compatible with the other VBC phases described above. In particular, for large (relative) $t_{6}$ the spectrum becomes quite dense at low energies preventing any VBC phase identification. ${ }^{15}$

In order to give a more quantitative determination of the region of stability of the mixed $\mathrm{CP}_{1}$ columnar-plaquette order, we have computed the related plaquette structure factors,

$$
I_{\beta}(q)=\frac{\left\langle\Psi_{0}\left|P_{\beta}(-q) P_{\beta}(q)\right| \psi_{0}\right\rangle}{\left\langle\Psi_{0} \mid \Psi_{0}\right\rangle},
$$

where $P_{\beta}(q)$ is a diagonal operator with the same symmetry as the degenerate GS listed in Table II that we aim to target, defined as Fourier transform of plaquette operators introduced in Ref. 10. For positive $t_{4}$ and $t_{6}$ values, such quantities can be computed efficiently using Green's-function quantum Monte Carlo (GFQMC). A Bragg peak of $I_{+}(q)$ at momentum $(\pi, \pi)(K$ point $)$ and a divergence of $I_{-}(q=0)(\Gamma$ point) reflect spontaneous translation and rotation symmetry breaking of the mixed phase, respectively. Related order parameters $M_{\beta}(q)=\sqrt{I_{\beta}(q)} / L$ can be conveniently defined and results are displayed in Fig. 3 showing size scalings of both $M_{+}(\pi, \pi)$ and $M_{-}(0,0)$ up to cluster size of 196 sites. These data correspond to the line $v / t_{4}=0.5$, i.e., originating from the expansion of the microscopic model $J_{1}-J_{2}-J_{3}$ under consideration here. Our results reveal that the Bragg peak at the $K$ point survives up to $\phi<0.3 \pi$, in good agreement with the ED criterion above. Interestingly, for increasing $\phi$, the columnar order parameter $I_{-}$vanishes before the plaquette one; hence rotation symmetry is first recovered and a narrow region of pure plaquette order is stabilized between the mixed phase region and the more complicated (unknown) phase at larger $t_{6}$. The extension of the GFQMC calculation to the $\theta$ $>0$ region which do not have ergodicity problems limitations, has enable to draw the phase diagram depicted in Fig. 4.

\section{CONCLUDING REMARKS}

To finish, let us summarize our findings and their implications. First we have extended the QDM to the case with a finite $t_{6}$ amplitude for the loop-6 kinetic processes. Although we have also extended the search for VBC phases, we found 
TABLE IV. (Color online) Expansion of $\mathcal{H}^{\text {eff }}$ up to order $\alpha^{8}$.

\begin{tabular}{|c|c|c|c|c|}
\hline \multirow{3}{*}{ Processes } & \multicolumn{4}{|c|}{$\mathcal{H}^{\text {eff }}=\mathcal{O}^{-1 / 2} \mathcal{H} \mathcal{O}^{-1 / 2}$} \\
\hline & Analytic & $J_{3}=J_{1} / 2$ & $J_{2}=J_{1} / 4$ & $J_{2}=J_{1} / 2$ \\
\hline & expression & $J_{2}=0$ & $J_{3}=J_{1} / 4$ & $J_{3}=0$ \\
\hline$\square$ & $2\left(J_{1}-J_{2}\right) \alpha^{4}\left(1+\alpha^{4}\right)$ & 0.625 & 0.46875 & 0.3125 \\
\hline$\square$ & $-2\left(J_{1}-J_{2}\right) \alpha^{2}\left(1+\alpha^{4}\right)$ & -1.25 & -0.9375 & -0.625 \\
\hline$\square$ & $\alpha^{4}\left(2\left(J_{3}-J_{1}+J_{2}\right)+\frac{1}{4} \alpha^{4}\left(5 J_{3}-J_{1}+J_{2}\right)\right)$ & -0.226562 & -0.242187 & -0.257812 \\
\hline$\square$ & $\left(-2 J_{1}+3 J_{2}+2 J_{3}\right) \alpha^{6}$ & -0.125 & -0.09375 & -0.0625 \\
\hline$\square$ & $\left(-J_{1}+J_{2}+2 J_{3}\right) \alpha^{6}$ & 0. & -0.03125 & -0.0625 \\
\hline$\| \square$ & $\left(J_{1}-J_{2}-J_{3}\right) \alpha^{6}$ & 0.0625 & 0.0625 & 0.0625 \\
\hline$\square$ & $\left(3 J_{1}-3 J_{2}-J_{3}\right) \alpha^{8}$ & 0.15625 & 0.125 & 0.09375 \\
\hline$\| \square$ & $\frac{1}{2}\left(-5 J_{1}+5 J_{2}+J_{3}\right) \alpha^{8}$ & -0.140625 & -0.109375 & -0.078125 \\
\hline $1+1$ & $4\left(J_{1}-J_{2}\right) \alpha^{8}$ & 0.25 & 0.1875 & 0.125 \\
\hline$\square^{-1}$ & $\frac{1}{4}\left(3 J_{1}-5 J_{2}-3 J_{3}\right) \alpha^{8}$ & 0.0234375 & 0.015625 & 0.0078125 \\
\hline$\square$ & $\frac{1}{4}\left(2 J_{1}-2 J_{2}-3 J_{3}\right) \alpha^{8}$ & 0.0078125 & 0.0117187 & 0.015625 \\
\hline$\square \square$ & $\frac{1}{4}\left(6 J_{1}-6 J_{2}-5 J_{3}\right) \alpha^{8}$ & 0.0546875 & 0.0507812 & 0.046875 \\
\hline$\square \square$ & $\frac{1}{4}\left(7 J_{1}-7 J_{2}-3 J_{3}\right) \alpha^{8}$ & 0.0859375 & 0.0703125 & 0.0546875 \\
\hline$\square$ & $\left(3 J_{1}-3 J_{2}+J_{3}\right) \alpha^{8}$ & 0.21875 & 0.15625 & 0.09375 \\
\hline$\square$ & $-\frac{1}{4}\left(3 J_{1}-5 J_{2}-6 J_{3}\right) \alpha^{8}$ & 0. & -0.00390625 & -0.0078125 \\
\hline$\square$ & $\frac{1}{4}\left(-5 J_{1}+9 J_{2}+6 J_{3}\right) \alpha^{8}$ & -0.03125 & -0.0195312 & -0.0078125 \\
\hline$\square$ & $\frac{1}{2}\left(3 J_{1}-J_{2}+4 J_{3}\right) \alpha^{8}$ & 0.15625 & 0.117187 & 0.078125 \\
\hline
\end{tabular}

that the previously known columnar, plaquette, and mixed (here called $\mathrm{CP}_{1}$ ) columnar-plaquette phases are stable when a moderate $t_{6}$ is added. This conclusion is first obtained by a close inspection of the low-energy spectrum of the model on finite clusters (ED). The quantitative determination of the extensions of the three previous phases is made possible by GFQMC simulations which do not suffer from the sign problem when $t_{6}>0$. Still, it has not been possible to characterize the GS in the whole parameter space, in particular, when $t_{6}$ dominates (accumulation of low-energy states) or when $v$ $<0$ (i.e., $\theta<0$ ) where the GFQMC encounters numerical limitations (both cases being physically irrelevant anyway). Our present work on the generalized QDM turns out to be very useful to make progress in the understanding of the frustrated $S=1 / 2$ quantum antiferromagnet on the square lattice. Indeed, it was previously argued that, in the vicinity of $J_{2} \simeq J_{3} \simeq J_{1} / 4$ and along the $J_{2}+J_{3}=J_{1} / 2$ line, a truncation in the NN SU(2)-singlet basis was legitimate. Using this argu- 
ment and generalizing the RK expansion in terms of the magnitudes of the overlaps of the elements of the truncated (nonorthogonal) basis we have made a link between the microscopic model and some small region of parameter space of the generalized QDM where, fortunately, a precise characterization of the phase can be made. We can therefore deduce that the frustrated $S=1 / 2$ quantum antiferromagnet exhibits in the vicinity of $J_{2} \simeq J_{3} \simeq J_{1} / 4$ the same type of lattice symmetry breaking as the mixed columnar-plaquette phase $\left(\mathrm{CP}_{1}\right)$ of the QDM. In the language of the quantum antiferromagnet, it is a eightfold degenerate $\mathrm{SU}(2)$-symmetric phase with a $2 \times 2$ unit cell (such as the plaquette phase) and rotation symmetry breaking (such as the columnar phase). While a previous investigation of the microscopic model on a $6 \times 6$ cluster has indeed provided evidence of plaquette correlations, ${ }^{7}$ only the mapping to the effective model (which can be studied on much larger clusters) provides enough accuracy to show the spatially anisotropic nature of this spinsinglet plaquette VBC phase.

\section{ACKNOWLEDGMENTS}

D.P. and A.R. are indebted to F. Becca for useful discussions at an early stage of this work. M.M. would like to thank D. Schwandt for his careful reading of the fusion rules.

\section{APPENDIX A: VARIATIONAL ANALYSIS}

The effective generalized quantum dimer model on the square lattice originated from the microscopic frustrated Heisenberg Hamiltonian studied here can be first investigated by a variational method. Indeed, it is possible to construct variational ansatze for valence-bond crystal phases (see inset of Fig. 5) as simple tensor products of resonating plaquette states, extending the number of possible phases arising in the standard QDM. Variational energies can then be computed analytically. Despite its simplicity, this approach reveals itself to be a useful guide for the numerical search of VBC (although artifacts due to its variational nature are expected) and can easily incorporate the symmetry analysis provided in the paper. In other words, there is a one-to-one correspondence between these variational wave functions (VWF) and the VBC states defined in the paper by the set of spontaneously broken symmetries.

We shall consider a set of five different VWF, (i) the well-known $|\mathrm{RK}\rangle$ one provided by Rokhsar and Kivelson ${ }^{9}$ as an equal weight superposition of all dimer configurations, (ii) three VWF based on four-site plaquette tensor products $\left(\left|\mathrm{CP}_{a}\right\rangle, a=1,2,3\right)$ and (iii) one with a six-site unit-cell interpolating between the columnar phase and the pure six-site plaquette phase (e.g., $|T\rangle$ in Fig. 5). Excepted for $|R K\rangle$, all these VWF depend on a parameter $\gamma$ which allows a continuous interpolation between pure singlet crystals and highly resonating VBC. To illustrate this construction, here is the expression of one of the anisotropic four-site plaquette phases and the above-mentioned six-site plaquette one,

$$
\begin{aligned}
\left|\mathrm{CP}_{1}\right\rangle & =\prod_{\otimes p} \cos (\gamma)|00\rangle_{p}+\sin (\gamma)|\mathrm{o}\rangle_{p} \\
|\mathrm{~T}\rangle & =\prod_{\otimes p^{\prime}} \sin (\gamma)|000\rangle_{p^{\prime}}+\frac{\cos (\gamma)}{\sqrt{2}}\left(|\mathrm{O} 0\rangle_{p^{\prime}}+|0 \&\rangle_{p^{\prime}}\right),
\end{aligned}
$$

where the product is performed over the set of separate plaquettes $p$ or $p^{\prime}$ as suggested in Fig. 5 .

In order to describe the stability of these phases w.r.t. the parameters, the expectation value of the effective generalized QDM Hamiltonian is computed. The only off-diagonal terms of $\mathcal{H}$ contributing to $\langle\Gamma|\mathcal{H}| \Gamma\rangle$, with $|\Gamma\rangle$ being one of those VWF, are plaquette flips on occupied plaquettes and the diagonal potential term. This leads to contributions proportional to $\cos (\gamma) \sin (\gamma)$ for all the VWF, plus one in $\cos ^{2}(\gamma)$ for the six-site plaquette one. For the diagonal terms, both occupied and nonoccupied plaquettes yield nonzero contributions to the expectation value of $\mathcal{H}$. It is worth to emphasize that the $|R K\rangle$ WF requires a special analysis. Using welldefined Pfaffian techniques, one can compute analytically the probability of flipping a four-site and a six-site plaquette, which are, respectively, $P_{4}=1 / 4$ and $P_{6}=0.0330112(1)$. Finally, these expectation values, as a function of the Hamiltonian parameters $v(\theta, \phi), t_{4}(\theta, \phi)$, and $t_{6}(\theta, \phi)$, are given by

$$
\begin{gathered}
E_{\mathrm{RK}}=v-t_{4}-4 t_{6} P_{6}, \\
E_{\mathrm{CP}_{1}}=v\left[1+\cos ^{4}(\gamma)+\sin ^{4}(\gamma)\right]-2 t_{4} \cos (\gamma) \sin (\gamma), \\
E_{\mathrm{CP}_{2}}=v\left[1+\cos ^{4}(\gamma)\right]-2 t_{4} \cos (\gamma) \sin (\gamma), \\
E_{\mathrm{CP}_{3}}=v\left[1+2 \cos ^{2}(\gamma) \sin ^{2}(\gamma)\right]-2 t_{4} \cos (\gamma) \sin (\gamma), \\
E_{T}=v\left[2 \cos ^{2}(\gamma)+4 \sin ^{2}(\gamma)+3 \cos ^{4}(\gamma) / 2+2 \sin ^{4}(\gamma)\right] / 3 \\
-t_{4} \frac{8 \cos (\gamma) \sin (\gamma)}{3 \sqrt{2}}-t_{6} \frac{2 \cos ^{2}(\gamma)}{3} .
\end{gathered}
$$

These energies are then minimized w.r.t. $\gamma$ and the variational phase diagram displayed in Fig. 5 as obtained in the $(\theta, \phi)$ plane. This phase diagram is discussed in the paper. The colors correspond to different values of the $\gamma$ parameter, $\gamma=\pi / 2$ (blue) for the pure columnar phase, $\gamma=0$ (purple) for the pure six-site resonating plaquette phase and $\gamma=\pi / 4$ (orange) for the isotropic four-site plaquette phase. The RK region has an arbitrary color.

\section{APPENDIX B: DERIVATION OF THE HAMILTONIAN}

This appendix is devoted to a technical description of the generalized QDM derivation scheme.

\section{Choice of a small parameter and disconnected processes}

As briefly described in the paper, the generalized QDM is obtained by developing $\mathcal{H}^{\text {eff }}=\mathcal{O}^{-1 / 2} \mathcal{H O}^{-1 / 2}$ according to the matrix element hierarchy of both $\mathcal{O}$ and $\mathcal{H}$ in the VB basis. Indeed, the amplitude of $\mathcal{O}_{\varphi, \psi}$ (with $\varphi$ and $\psi$ two VB configurations) only depends on the number of loops $n_{l}$ of the overlap diagram $g=(\varphi, \psi)$ obtained by superimposing the 


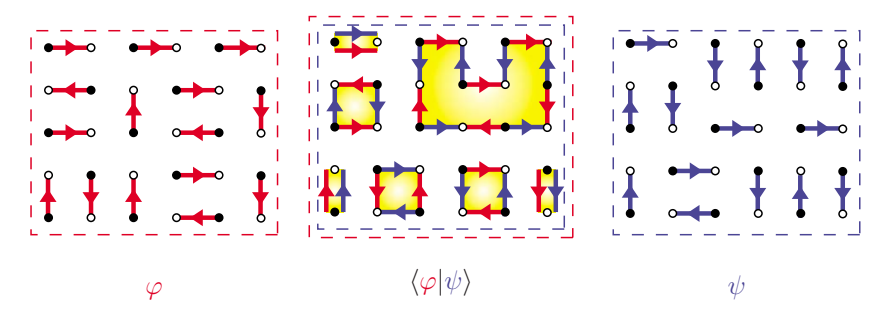

FIG. 6. (Color online). Overlap $\langle\varphi \mid \psi\rangle$ between two VB configurations $\varphi$ ad $\psi$. Closed loops that appear in the overlap diagram $g$ are represented as yellow shades.

two configurations: $\mathcal{O}_{g}=\alpha^{N-2 n_{l}(g)}$ with $\alpha=1 / \sqrt{2}$ and $N$ the total number of sites (see Fig. 6).

The maximal number of loops is obtained for $\varphi=\psi$. In this case $g$ is just a collection of $N / 2$ trivial loops with length $\mathcal{L}_{l}=2$ and $\mathcal{O}_{g}=1$. The next term is obtained by considering one nontrivial loop with length $\mathcal{L}_{l}=4(\square) \quad$ while all remaining $(N-4) / 2$ loops are chosen trivial which leads to $\mathcal{O}_{g}=\alpha^{2}$. In the same spirit, one $\mathcal{L}_{l}=6 \operatorname{loop}(\square)$ and $(N-6) / 2$ trivial loops is an $\alpha^{4}$-order process (see the fourth line of Tables I and III). Such a construction suggests that a quite natural driving parameter for the expansion is the length $\mathcal{L}_{l}$ of a unique nontrivial loop surrounded by $\left(N-\mathcal{L}_{l}\right) / 2$ trivial loops: such a process indeed appears at order $\alpha^{\mathcal{L}_{l}-2}$. However the total length of loops is a constraint quantity $\left(\Sigma_{l \in g} \mathcal{L}_{l}=N\right)$ and other (nonconnected) terms indeed appear in $\mathcal{O}$ at the same order.

For example, $\square \square$ formed by two disconnected squares also occurs in $\mathcal{O}$ with the amplitude $\alpha^{4}$ despite the fact the nontrivial contour length is different from, e.g.,

For this reason, in the derivation scheme presented here, we chose the overall number of loops as the driving parameter for the expansion of $\mathcal{O}$ rather than the commonly used length of the loops. ${ }^{9,12,13}$ The key difference lies in the presence of disconnected processes such as $\square \square$ : as we will see, they cancel at every order of the final effective Hamiltonian but are crucial in the calculation because they are responsible for the emergence of nontrivial diagonal and off-diagonal connected processes.

In the expansion of $\mathcal{O}$, we use the following notation:

$$
\mathcal{O}=\sum_{p \geq 0} \alpha^{2 p} \omega_{p}
$$

where $\omega_{p}$ are combinations of $\omega_{p}^{g}$ process on graphs $g$,

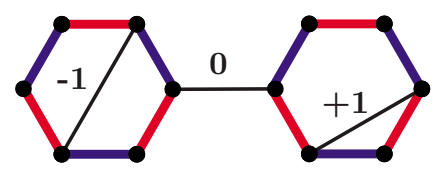

FIG. 7. (Color online). The matrix element $\left\langle\varphi\left|(4 / 3) \mathbf{S}_{i} . \mathbf{S}_{j}\right| \psi\right\rangle$ expressed as $f_{i j}\langle\varphi \mid \psi\rangle$. Bond $(i, j)$ is represented as a solid black line. Red and blue bonds represent $|\varphi\rangle$ and $|\psi\rangle$. $f_{i j}=+1$ (respectively, $f_{i j}=-1$ ) if $i$ and $j$ belong to the same loop at even (respectively odd) distance along the loop. $f_{i j}=0$ if $(i, j)$ connects two sites on distinct loops (see Ref. 16).

$$
\omega_{p}=\sum_{g} \omega_{p}^{g} .
$$

For a full list of $\omega_{p}^{g}$ up to order $\alpha^{8}$, see Table III.

\section{Heisenberg Hamiltonian expansion}

The action of each term of the Heisenberg Hamiltonian (1) of a VB state consist in a reconfiguration of dimers. Thus, $\langle\varphi|\mathcal{H}| \psi\rangle$ can be deduced form inspecting the topology of the overlap diagram $\langle\varphi \mid \psi\rangle$ as recalled in Fig. 7. This allow $\mathcal{H}$ to be expanded in power of $\alpha$ (see Ref. 16) similarly to $\mathcal{O}$,

$$
\mathcal{H}=\sum_{p \geq 0} \alpha^{2 p} \sum_{g} h_{p}^{g} \omega_{p}^{g} .
$$

Note that it is convenient here to rescale the Hamiltonian by a factor $4 / 3$. Furthermore, evaluating $\left\langle\varphi\left|(4 / 3) \mathbf{S}_{i} . \mathbf{S}_{j}\right| \psi\right\rangle$ generically involves an extensive number of length-2 loops which only effect is to produce a trivial extensive contribution to the matrix element. This can be removed by scaling and shifting $\mathcal{H}$ to $(4 / 3) \mathcal{H}+J_{1} N / 2$. Using this convention, the expansion of $\mathcal{H}$ contains only kinetic terms. For a full list of $h_{p}^{g}$ up to order $\alpha^{8}$, see the last column of Table III.

\section{Fusion rules and effective Hamiltonian}

The first step to compute the effective Hamiltonian $\mathcal{H}^{\text {eff }}$ $=\mathcal{O}^{-1 / 2} \mathcal{H O}^{-1 / 2}$ is to derive the expression of $\mathcal{O}^{-1 / 2}$. To achieve this, we use the formal expansion,

$$
\mathcal{O}^{\tau}=\sum_{k \geq 0} \frac{\Gamma(1+\tau)}{\Gamma(1+\tau-k) \Gamma(1+k)}(\mathcal{O}-1)^{k} .
$$

Powers of $\mathcal{O}$ and products with $\mathcal{H}$ generically involve symmetric products of diagrams (see Table III) that do not commute, e.g,

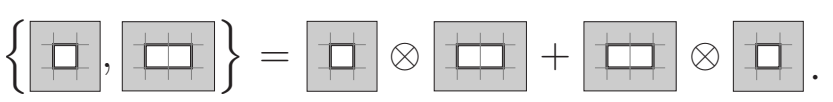

Evaluating these products requires establishing fusion rules that (i) governs algebraic properties of diagrams and (ii) generate, order by order, extra diagonal and off-diagonal processes. We give below, the minimal set of rule that are needed up to order $\alpha^{8}$.

Order 4 fusion rule,

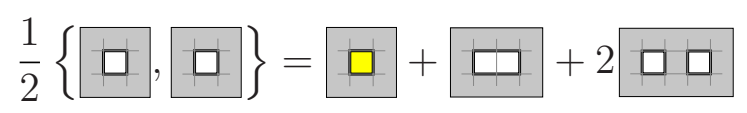


Order 6 fusion rules,

$$
\begin{aligned}
& \{\square, \square \square\}=2 \square \square+6 \square \square \square+2 \square \square+\square \\
& \{\square, \square\}=2 \square+2 \square \square+\square \square \\
& \{\square, \square\}=1 \square+2 \square \square+2 \square+2 \square
\end{aligned}
$$

Order 8 fusion rules,

$$
\begin{aligned}
& \frac{1}{2}\{\square, \square\}=\square+2 \square \square+2 \square \\
& \{\square, \square\}=2 \square \square+\square+\square \\
& \{\square, \square \square\}=4 \square \square+2 \square \square \square+1 \square \square+\square \square \\
& \frac{1}{2}\{\square \square, \square \square\}=\square \square+2 \square \square \square+\square \square+6 \square \square \square \square \\
& + 2 \longdiv { \square \square \square } + \square \square + \square + \square \\
& \frac{1}{2}\{\square, \square, \square\}=\square+\square+\square+\square+\square \\
& +\square+\square \\
& \{\square \square, \square\}=\square \square \square+\square+2 \square \square \square+2 \square \square \\
& +2 \square \square+\square+\square+\square+\square \\
& \{\square, \square \square \square\}=2 \square \square \square+8 \square \square \square \square+2 \square \square \square+\square \square \\
& \{\square, \square \square\}=1 \square \square+2 \square \square+4 \square \square \square+4 \square \square \\
& + 2 \longdiv { \square \square } + 2 \square + 2 \square + \square \\
& \{\square, \square\}=\square+2 \square+\square \\
& +2 \square+\square+\square \\
& \{\square, \square\}=\square+2 \square+2+\square \\
& \{\square, \square \square\}=4 \square \square+4 \square \square+4 \square \square \square+\square \square \\
& +2 \square \square+\square
\end{aligned}
$$

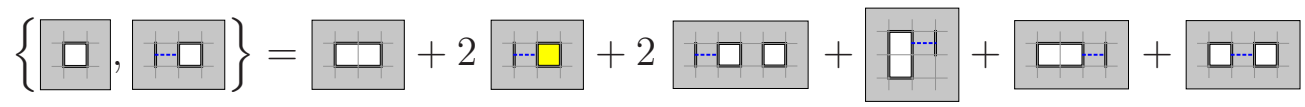

$$
\begin{aligned}
& \square \square=\square
\end{aligned}
$$


Note that contractions of diagrams not only produce potential (diagonal) terms but also nontrivial assisted off-diagonal operators such as, e.g., $\quad+-\square$ that flip a plaquette only if a dimer is present next to it. Special process also appear such as $\square \square$ (respectively $\square \square$ ) which simultaneously

flip two neighboring plaquettes with parallel (respectively, perpendicular) dimers. In Table IV, we summarize the result of the expansion up to order $\alpha^{8}$. Interestingly enough, all disconnected terms vanish after simplifying the product $\mathcal{O}^{-1 / 2} \mathrm{HO}^{-1 / 2}$. The demonstration of this generic property is beyond the scope of the present paper and will be presented elsewhere. ${ }^{17}$ At this level, let us remark that this absence of nonlocal terms in the generalized QDM Hamiltonian is physically satisfactory and is a strong indication of the internal consistency of the derivation scheme presented here.
${ }^{1}$ G. Chaboussant, M.-H. Julien, Y. Fagot-Revurat, M. Hanson, L. P. Lvy, C. Berthier, M. Horvati, and O. Piovesana, Eur. Phys. J. B 6, 167 (1998).

${ }^{2}$ P. Mendels, F. Bert, M. A. de Vries, A. Olariu, A. Harrison, F. Duc, J. C. Trombe, J. Lord, A. Amato, and C. Baines, Phys. Rev. Lett. 98, 077204 (2007).

${ }^{3}$ S. Miyahara and K. Ueda, J. Phys.: Condens. Matter 15, R327 (2003).

${ }^{4}$ E. Chappel, D. Nuñez-Regueiro, G. Chouteau, O. Isnard and C. Darié, Eur. Phys. J. B 17, 615 (2000).

5 J. Robert, V. Simonet, B. Canals, R. Ballou, E. Lhotel, C. Darie, P. Bordet, B. Ouladdiaf, M. Johnson, J. Ollivier, D. Braithwaite, H. Rakoto, and S. de Brion, Phys. Rev. B 77, 054421 (2008).

${ }^{6}$ P. W. Anderson, Science 235, 1196 (1987).

${ }^{7}$ M. Mambrini, A. Lauechli, D. Poilblanc, and F. Mila, Phys. Rev. B 74, 144422 (2006), and references therein. See also recent work using projected entangled paired states (PEPS); V. Murg, F. Verstraete, and J. Cirac, ibid. 79, 195119 (2009).

${ }^{8} \mathrm{~A}$ valence bond is a (spatially antisymmetric) singlet pair of two $\mathrm{S}=1 / 2$ spins.

${ }^{9}$ D. S. Rokhsar and S. A. Kivelson, Phys. Rev. Lett. 61, 2376
(1988).

${ }^{10}$ A. Ralko, D. Poilblanc, and R. Moessner, Phys. Rev. Lett. 100, 037201 (2008)

${ }^{11}$ S. Wessel, Phys. Rev. B 78, 075112 (2008); F. Trousselet, D. Poilblanc, and R. Moessner, ibid. 78, 195101 (2008).

${ }^{12}$ D. Poilblanc, F. Alet, F. Becca, A. Ralko, F. Trousselet, and F. Mila, Phys. Rev. B 74, 014437 (2006); A. Ralko, F. Mila, and D. Poilblanc, Phys. Rev. Lett. 99, 127202 (2007).

${ }^{13}$ R. Moessner and S. L. Sondhi, Phys. Rev. Lett. 86, 1881 (2001); F. Vernay, A. Ralko, F. Becca, and F. Mila, Phys. Rev. B 74, 054402 (2006)

${ }^{14}$ For reviews see, e.g., H. J. Schulz, T. Ziman, and D. Poilblanc, in Magnetic Systems with Competing Interactions, edited by H. T. Diep (World Scientific, Singapore, 1994), pp. 120-160; G. Misguich and C. Lhuillier, in Frustrated Spin Systems, edited by H. T. Diep (World Scientific, Singapore, 2005).

${ }^{15}$ Note that the trimerized phase is frustrated on the $8 \times 8$ cluster. However, investigation of $6 \times 6$ and $6 \times 10$ clusters have not revealed any clear sign of its stability either.

${ }^{16}$ B. Sutherland, Phys. Rev. B 37, 3786 (1988).

${ }^{17}$ D. Schwandt, M. Mambrini, and D. Poilblanc (unpublished). 